Sir,

\section{Bilateral dacroadenitis associated with acute Crohn's disease}

Crohn's disease is an idiopathic inflammatory disease of the upper and lower intestinal tract characterized by transmural inflammatory ulcers of the digestive system featuring noncaseating granulomas. Extraintestinal manifestations are present in 25-35\% of patient with Crohn's disease. Ocular manifestations occur in less than $10 \%$ of cases and include episcleritis, scleritis, uveitis, retinal vasculitis, optic neuropathy, and idiopathic orbital inflammatory disease. ${ }^{1-3}$

We report the case of a 10-year-old female who presented with bilateral dacroadenitis as one of the initial manifestations of previously unrecognized Crohn's disease.

\section{Case report}

A previously healthy 10-year-old Caucasian female was referred to the eye department with a 1-week history of bilateral upper eyelid swelling associated with moderate discomfort. The patient had a 3-weeks history of fever, abdominal pain, diarrhoea, aphthous oral lesions, and arthritis.

Examination disclosed uncorrected visual acuity of 20/20 OU, and bilateral upper eye lid swelling, more prominent temporally, conferring an ' $\mathrm{S}$ '-shaped contour to the margin (Figure 1). The upper eyelids were tender, and erythematous with a light purple hue. Bilateral mild lacrimal gland swelling was present. There was no evidence of proptosis or ocular motility abnormalities. Examination of the anterior segment, retina, and optic nerve was unremarkable. A diagnosis of bilateral dacroadenitis was made.

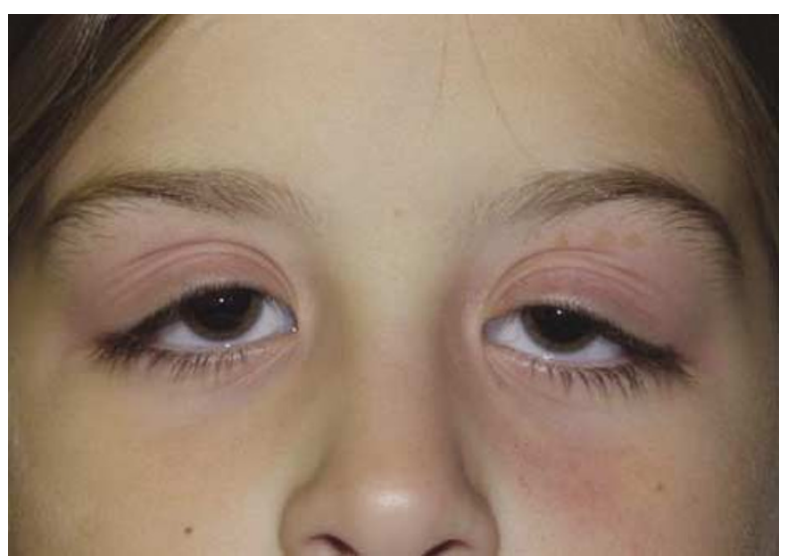

Figure 1 Bilateral upper eyelid swelling, more prominent temporally, conferring an ' $\mathrm{S}$ '-shaped contour to the margin. The skin was erythematous and had a light purple hue. Lacrimal gland swelling was present bilaterally.
Laboratory investigation revealed leukocytosis, anaemia, and an ESR of $70 \mathrm{~mm} / \mathrm{h}$. Autoantibody screen, and serology for Epstein-Barr virus were negative. Colonoscopy disclosed multiple deep ulcers throughout the colon. Histopathologic examination of biopsied specimen demonstrated granulomatous inflammation involving the terminal ileum and colon, confirming the diagnosis of Crohn's disease.

The patient was treated with intravenous methyl prednisolone $(1.6 \mathrm{mg} / \mathrm{kg} /$ day) for 3 days followed by oral therapy. Her symptoms responded promptly to the treatment with complete resolution of dacroadenitis within 2 weeks.

\section{Comment}

Crohn's disease is an immune-based systemic disorder that predominantly involves the gastrointestinal tract. Extraintestinal manifestations often correlate with the disease activity and may precede, occur concurrently, or follow the initial gastrointestinal presentation of the disease. Ocular complications occur in approximately $10 \%$ of patients with Crohn's disease and include conjunctivitis, keratitis, episcleritis, scleritis, uveitis, retinal haemorrhage and oedema, retinal vasculitis, neuroretinitis, optic neuropathy, orbital myositis, and neuro-ophthalmologic abnormalities. ${ }^{1-5}$ Dacroadenitis is rarely associated with Crohn's disease. ${ }^{4,5}$ The presence of one or more extraintestinal complications significantly increases the chance of ocular involvement. Patients with Crohn's disease affecting the colon or ileocolon are more likely to have ocular inflammation than those with small bowel involvement. Medical or surgical treatment of gastrointestinal inflammation frequently results in the improvement of the ophthalmic complications.

Orbital myositis and idiopathic orbital inflammatory disease may occur in association with Crohn's disease. Our patient did not exhibit any symptoms or signs suggestive of these conditions and it appears that the lacrimal gland was the primary orbital tissue that was clinically affected. Other causes of dacroadenitis were ruled out by clinical and laboratory findings in our patient.

In summary, we report a case of bilateral dacroadenitis that presented concurrently with the first manifestations of Crohn's disease. The patient received systemic corticosteroid therapy with rapid resolution of dacroadenitis, and the inflammatory bowel disease.

\section{References}

1 Hopkins DJ, Horan E, Burton IL, Clamp SE, deDombal FT, Goligher JC. Ocular disorders in a series of 332 patients with Crohn's disease. Br J Ophthalmol 1974; 58: 732-737. 
2 Knox DL, Schachat AP, Mustonen E. Primary, secondary and coincidental ocular complications of Crohn's disease. Ophthalmology 1984; 91: 163-173.

3 Salmon JF, Wright JP, Murray ADN. Ocular inflammation in Crohn's disease. Ophtalmology 1991; 98: 480-484.

4 Dutt S, Cartwright MJ, Nelson CC. Acute dacryoadenitis and Crohn's disease: findings and management. Ophthal Plast Reconstr Surg 1992; 8: 295-299.

5 Hwang IP, Jordan DR, Acharya V. Lacrimal gland inflammation as the presenting sign of Crohn's disease. Can J Ophthalmol 2001; 36: 212-213.

N Rafiei ${ }^{1}, \mathrm{H}$ Tabandeh$^{1}$ and M Hirschbein ${ }^{2}$

${ }^{1}$ The Wilmer Eye Institute, Baltimore, MD, USA

${ }^{2}$ The Krieger Eye Institute, Sinai Hospital, Baltimore, MD, USA

Correspondence: H Tabandeh, Department of Ophthalmology, Wilmer Eye Institute, B-20, Johns Hopkins Hospital, 600 N. Wolfe Street, Baltimore, MD 21287-9248, USA

Tel: + 1410955 8265;

Fax: + 14106148496

E-mail: htaband1@jhmi.edu

Eye (2006) 20, 1372-1373. doi:10.1038/sj.eye.6702205;

published online 9 December 2005

Sir,

The surgical management of chronic hypotony due to uveitis

Prolonged hypotony in uveitis patients is often regarded as the end stage of a chronic disease from which recovery is improbable if not impossible. However, not all hypotony cases are alike. Hypotony resulting from active inflammation will respond to adequate immunosuppression, and as indicated in our article an attempt should be made to treat it medically before considering a surgical approach. The question then is how long should one wait to observe a response.

As indicated by Dr Liu and co-workers, periocular steroids can have a prolonged effect. In certain forms of uveitis, a single periocular injection can provide a beneficial effect for 8-12 weeks. However, one would expect to see a response to steroids within the first 10-14 days. To take into account a possible delay in this initial response, we followed patients for 2 months prior to surgery. The patients included in this series did not show a pressure rise on intensified immunosuppression.
There is no doubt that steroids and other immunosuppressants contribute to the surgical result. However, the effect will not be sustained once a taper is initiated. Management in this series of patients became easier following surgery with fewer and less immunosuppressants being required. Hypotony in chronic uveitis patients is often characterized by a protracted course requiring frequent reinjections, or modifications to the immunosuppressive regimen.

For all the reasons mentioned above, and our results, we feel that a surgical approach should be considered in this group of patients. With time, we should be able to determine the place and timing of surgery in the management of this severe complication of uveitis.

MD de Smet, F Gunning and R Feenstra

Department of Ophthalmology, University of

Amsterdam, Meibergdreef 9, Rm G2-217,

Amsterdam 1105 AZ, The Netherlands

Correspondence: MD de Smet,

Tel: + 3120566 3455;

Fax: + 31205669053 .

E-mail: m.d.desmet@amc.uva.nl

Eye (2006) 20, 1373. doi:10.1038/sj.eye.6702208;

published online 9 December 2005

Sir,

The surgical management of chronic hypotony due to uveitis

Dr de Smet and associates have conducted an interesting study on surgical interventions for cases of uveitisinduced chronic hypotony. After a joyous reading of the whole article, we think that an important issue should warrant further discussion.

Subtenon's capsule triamcinolone acetonide injection was shown to be effective in the management of intraocular inflammation. ${ }^{1,2}$ It has an overt advantage over systemic steroid for effaced systemic adverse effect and slow-releasing depot. ${ }^{1}$ The biological action of subtenon triamcinolone acetonide is long and can be up to 6 weeks or even longer. ${ }^{1,2}$

From the methodology, it can be learned that some of the patients with intraocular inflammation were given one to two subtenon's injection prior to the surgical intervention. ${ }^{3}$ Interestingly, if one inspected Table 1 of the article, it was noted that duration of hypotony in patient numbers 1-4 ranged from 8 to 12 weeks. ${ }^{3}$ Apparently, 